\title{
PAK5 overexpression is associated with lung metastasis in osteosarcoma
}

\author{
KUN HAN $^{1 *}$, YAN ZHOU $^{1 *}$, KUO-FU TSENG ${ }^{2}$, HAIYAN HU $^{1}$, KUNPENG LI $^{3}$, \\ YALING WANG $^{1}$, ZHIHUA GAN ${ }^{1}$, SHUCHEN LIN ${ }^{1}$, YONGNING SUN ${ }^{1}$ and DALIU MIN ${ }^{1}$ \\ ${ }^{1}$ Oncology Department, Shanghai Jiao Tong University Affiliated Sixth People's Hospital, Shanghai 200233, P.R. China; \\ ${ }^{2}$ Biophysics Department of Oregon State University, Corvallis, OR 97330, USA; ${ }^{3}$ School of Life Sciences, \\ Sun Yat-Sen University, Guangzhou, Guangdong 510275, P.R. China
}

Received September 18, 2015; Accepted February 23, 2017

DOI: $10.3892 / \mathrm{ol} .2017 .7545$

\begin{abstract}
PAKs) are multifunctional effectors of Rho GTPases, which are associated with cytoskeletal organization, cellular morphogenesis, migration and survival. PAKs are overactive in a number of tumor tissues and have attracted attention as a potential target for cancer therapy. In the present study, PAK5 levels were analyzed in primary osteosarcoma (OS) samples $(n=65)$ using reverse transcription-quantitative polymerase chain reaction (RT-qPCR) and immunohistochemistry (IHC) methods. In the primary OS tissue, increased PAK5 expression (IHC score $>2, n=37$ ) was associated with significantly decreased overall survival $(\mathrm{P}=0.036)$ compared with decreased PAK5 expression (IHC score $\leq 2, n=28)$. PAK5 expression was identified to be significantly associated with metastasis $(\mathrm{P}=0.010)$. The lung is the most common metastasis site for OS. In addition, the level of PAK5 in lung metastasis tissue $(n=13)$ was detected using RT-qPCR and IHC methods. PAK 5 expression was increased in lung metastasis tissue compared with in primary OS samples. PAK5 was silenced using short hairpin RNA in OS cell lines. Wound healing, migration and nude mice model assay results consistently demonstrated that PAK5 knockdown was able to significantly inhibit OS migration. In PAK5-knockdown cells, the alteration in the expression of a number of metastasis-associated factors, including epithelial cadherin, vimentin, fibronectin and matrix metalloproteinase 2 (MMP2), was analyzed. Only MMP2 expression was decreased significantly $(\mathrm{P}<0.05)$. The expression level of MMP2 was analyzed in
\end{abstract}

Correspondence to: Dr Yongning Sun or Dr Daliu Min, Oncology Department, Shanghai Jiao Tong University Affiliated Sixth People's Hospital, 600 Yishan Road, Shanghai 200233, P.R. China

E-mail: ynsun2002@126.com

E-mail: mindaliu@csco.org.cn

*Contributed equally

Key words: p21-activated kinase 5, osteosarcoma, lung metastasis, matrix metalloproteinase 2 primary OS tissue and lung metastasis tissue using RT-qPCR and IHC methods. Expression of MMP2 was identified to be associated with expression of PAK5. The results of the present study suggest that PAK5 promotes OS cell migration and that PAK5 expression may be used to predict lung metastasis.

\section{Introduction}

Osteosarcoma (OS), the most common primary malignant bone tumor in children and adolescents, generally originates from the metaphysis of the long bone and has a high propensity for local invasion and distant metastases, most commonly the lung (1-3). In spite of aggressive surgery, intensive chemotherapy and multidisciplinary treatment, the survival rate of OS has remained constant over the last few decades (4-6). The long-term survival rate of patients with metastasis or recurrence remains at $<20 \%$ (7). The metastatic biology of OS is now beginning to be understood, which holds promise for the development of novel strategies to improve survival rates in patients with OS.

The p21-activated kinases (PAKs) are a family of Rac- $\alpha$ serine/threonine protein kinase $(\mathrm{Rac}) /$ cell division cycle 42 (Cdc42)-associated serine/threonine protein kinases that are characterized by a highly-conserved $\mathrm{N}$-terminal Cdc42/Rac-interactive binding domain and a C-terminal kinase domain (8). PAKs serve important roles in regulating a variety of cellular functions, including cytoskeletal dynamics (9), morphology, cell survival (10), migration (11) and mitosis (12). The six human PAK isoforms are classified into two families on the basis of their amino acid sequence and function: Group I PAKs (PAK1, PAK2 and PAK3) exhibit between 80 and $90 \%$ sequence identity within their catalytic domains, whereas group II PAKs (PAK4, PAK5 and PAK6) exhibit between 40 and $50 \%$ sequence identity with the kinase domains of the group I PAKs (13).

PAK5, the most recently discovered PAK family member, exhibits unique characteristics that differentiate it from the other PAKs, and its expression was primarily identified in neuronal tissues (14). Unlike PAK1, PAK5 has no ability to complement serine/threonine protein kinase STE20 function, and its high basal activity is not regulated by GTPases, although it is able to bind to Cdc42 in preference to Rac (15). 
Furthermore, PAK5 localizes to the mitochondria, protects cells from pro-apoptotic stimuli by phosphorylating B-cell lymphoma 2-associated death promoter (Bad) on Ser-112 and inhibits apoptosis by preventing Bad from entering the mitochondria (15). PAK5 is reportedly overexpressed in pancreatic cancer (16), colorectal cancer (17), gastric cancer (18), breast cancer (19) and glioma (20), and its potential ability to promote tumor behavior in carcinogenesis has been investigated. However, the underlying molecular mechanisms of PAK5 in OS progression remain unclear.

In the present study, we identified that PAK5 expression was upregulated in OS tissue and OS cell lines. PAK5 expression was significantly increased in OS patients with metastasis. Therefore, the effect and underlying molecular mechanism of PAK5 in OS progression was investigated. The results of the present study suggested that PAK5 knockdown is able to inhibit cell migration and invasion in human OS cell lines. Matrix metalloproteinase 2 (MMP2), a downstream signaling molecule, was identified to participate in the regulation of the process. Therefore, it is proposed that PAK5 may serve an essential role in OS development and that PAK5 is a potential therapeutic target.

\section{Materials and methods}

Tissue samples of patients with OS. The present study was a retrospective analysis. Owing to loss to follow-up, 2 years was selected as the survival duration. A total of 65 patients with OS who underwent surgery were enrolled. Among them, 13 exhibited lung metastasis. Primary tumor tissue and pulmonary metastasis tissue were collected to analyze the expression of PAK5 and MMP2 at the mRNA and protein levels using the reverse transcription-quantitative polymerase chain reaction (RT-qPCR) and immunohistochemistry (IHC). The samples were collected from Shanghai Jiao Tong University Affiliated Sixth People's Hospital (Shanghai, China) from January 2009 to January 2012. The patients' clinical characteristics included age at diagnosis, sex, tumor size, clinical stage, pathological type, necrosis rate and metastasis. The present study was conducted following approval from the Ethics Committee of Shanghai Jiao Tong University Affiliated Sixth People's Hospital and written informed consent was provided by all patients.

Immunohistochemistry. Primary osteosarcoma tissue and pulmonary metastasis tissue, which were paraffin-embedded, were sliced into $4-\mu \mathrm{m}$ sections. Paraffin-embedded tissue slides were heated for $45 \mathrm{~min}$ at $60^{\circ} \mathrm{C}$, deparaffinized in xylene, rehydrated in alcohol gradient and then non-specifically blocked using $\mathrm{H}_{2} \mathrm{O}_{2}$ for $10 \mathrm{~min}$ and non-specific serum (Maixin-Bio, Fuzhou, China) for $15 \mathrm{~min}$ at room temperature subsequent to antigens being exposed with citric acid buffer (Maixin-Bio) ( $\mathrm{pH} 6.0 ; 0.1 \mathrm{M}$ ) and heated by microwave for $10 \mathrm{~min}$. The paraffin sections were incubated with primary antibody against PAK5 (catalog no., ab110069) and MMP2 (catalog no., ab37150) (dilution, 1:50; Abcam, Cambridge, UK). Rabbit IgG (dilution, 1:100; catalog no., ab172730, Abcam) was used as a negative control. Immunohistochemical scoring was performed without prior knowledge of the clinical response. Tissue sections were observed using an AX10-Imager A1 light microscope (Zeiss GmbH, Jena, Germany), and all images were captured using AxioVision microscopy software (version 4.7; Zeiss GmbH). The tissue sections were then manually scored semi-quantitatively for cytoplasmic staining. The positive particles appeared brown in the cytoplasm. The dominant staining intensity in tumor cells was scored as: 0 , $0 \%$; 1 , between 1 and $25 \%$; 2, between 26 and 50\%; 3, between 51 and $75 \%$; and 4, between 76 and $100 \%$.

Cell culture. The human OS cell lines Saos-2 and MG63 were obtained from the American Type Culture Collection (Manassas, VA, USA). Cells were cultured in Dulbecco's modified Eagle's medium (DMEM; Gibco; Thermo Fisher Scientific, Inc., Waltham, MA, USA) supplemented with $10 \%$ fetal bovine serum (FBS; Gibco; Thermo Fisher Scientific, Inc.) and 100 units penicillin/streptomycin (Invitrogen; Thermo Fisher Scientific, Inc.) and maintained at $37^{\circ} \mathrm{C}$ in a humidified atmosphere containing $5 \% \mathrm{CO}_{2}$.

Construction of recombinant lentivirus and lentivirus infection. The cDNA sequence of PAK5 was obtained from GenBank $^{\circledR}$ (www.ncbi.nlm.nih.gov/genbank; accession number NM_177990). The PAK5 short hairpin RNA (shRNA) 5'-CTA GCCGGGATTACCACCATGACAATTTCAAGAGAATTGT CATGGTGGTAATCCCGTTTTTTGGAATTAAT-3') that targets nucleotides 1,934-1,954 of the PAK5 mRNA was inserted into the plasmid pFH-L (Shanghai Hollybio, Shanghai, China). Non-silencing shRNA (5'-CTAGCCCGGTTCTCC GAACGTGTCACGTATCTCGAGATACGTGACACGTTCG GAGAATTTTTTTAAT-3') was used as a control that does not target any genes in humans, mice or rats as determined by NCBI Reference Sequence Database (www.ncbi.nlm.nih. gov/refseq) screening. The recombinant vector was confirmed by digestion analysis using restriction endonucleases (NotI and Bam HI), and all inserted sequences were verified by DNA sequencing by external company (Invitrogen; Thermo Fisher Scientific, Inc.). The lentiviruses were generated by triple transfection of $80 \%$ confluent human embryonic kidney HEK-293 cells with PAK5 (or control) shRNA-expressing vector and the virion-packaging elements (pVSVG-I and pCMV $\Delta$ R8.92) using Lipofectamine $^{\mathrm{TM}} 2000$ (Invitrogen; Thermo Fisher Scientific, Inc.). The lentiviruses were harvested in serum-free medium after 3 days and filtered through a $0.45-\mu \mathrm{m}$ filter (EMD Millipore, Billerica, MA, USA).

Saos-2 and MG63 cells were plated at $5 \times 10^{4}$ cells/well in 6 -well plates with recombinant lentivirus-encoding shRNA against PAK5 (Lv-shPAK5) at a multiplicity of infection of 60 in serum-free DMEM at $37^{\circ} \mathrm{C}$. After $2 \mathrm{~h}$, serum-enriched DMEM was added to the cells, and the growth medium was replaced after $24 \mathrm{~h}$. At 3 days post-transfection, reporter gene expression (green fluorescent protein) was examined using fluorescence microscopy.

$R T-q P C R$. Total cellular RNA from Saos-2 and MG63 cells was isolated using TRIzol ${ }^{\circledR}$ reagent (Invitrogen; Thermo Fisher Scientific, Inc.) and amplified using an RT-qPCR kit (Platinum ${ }^{\circledR}$ SYBR $^{\circledR}$ Green qPCR SuperMix-UDG; Applied Biosystems; Thermo Fisher Scientific, Inc.), according to the manufacturer's protocol. A $1 \mu \mathrm{g}$ sample of total RNA was used for first-strand cDNA synthesis using Moloney murine 
leukemia virus reverse transcriptase at the following temperature protocol: $5 \mathrm{~min}$ at $25^{\circ} \mathrm{C} ; 60 \mathrm{~min}$ at $42^{\circ} \mathrm{C}$; and $5 \mathrm{~min}$ at $70^{\circ} \mathrm{C}$. The primer sequences used for PAK 5 were $5^{\prime}-\mathrm{GGC}$ GTCCTCTTGTGTCTTC-3' (forward) and 5'-GTACTGAGT CCTTCTGATTTGC-3' (reverse). The primer sequences for MMP2 were: 5'-CTTCTTCCCTCGCAAGCC-3' (forward) and 5'-ATGGATTCGAGAAAACCG-3' (reverse). $\beta$-actin was used as the internal control and amplified with the primers 5'-GTGGACATCCGCAAAGAC-3' (forward) and 5'-AAA GGGTGTAACGCAACTA-3' (reverse). qPCR was conducted using the CFX Connect ${ }^{\mathrm{TM}}$ Real-Time PCR Detection system (Bio-Rad Laboratories, Inc., Hercules, CA, USA). The qPCR conditions were as follows: $2 \mathrm{~min}$ at $50^{\circ} \mathrm{C} ; 10 \mathrm{~min}$ at $95^{\circ} \mathrm{C}$; 35 cycles of $10 \mathrm{sec}$ at $95^{\circ} \mathrm{C}$; and $1 \mathrm{~min}$ at $60^{\circ} \mathrm{C}$. All reactions were performed in triplicate. Relative expression levels of PAK5 in non-transduced and transduced Saos-2 and MG63 cells were determined using the $2^{-\Delta \Delta \mathrm{Cq}}$ method by Microsoft Excel 2007 (21).

Western blot analysis. The non-transduced and transduced Saos- 2 and MG63 cells were lysed in $50 \mu 1$ lysis buffer $(100 \mathrm{mM}$ Tris- $\mathrm{HCl}, \mathrm{pH}$ 6.8, 10 mM EDTA, 4\% SDS and 10\% glycine) on ice for $15 \mathrm{~min}$. The lysates were clarified by centrifugation at $12,000 \mathrm{x} \mathrm{g}$ for $30 \mathrm{~min}$ at $4^{\circ} \mathrm{C}$. The supernatants were collected and protein concentrations were determined using the bicinchoninic acid method. Equal amounts (30 $\mu \mathrm{g}$ protein) of lysate were subjected to SDS-PAGE (12\% gels) prior to being transferred onto a polyvinylidene fluoride membrane using an electroblotting apparatus. The membrane was blocked for $1 \mathrm{~h}$ at room temperature with 5\% non-fat dried milk in TBS and Tween-20 (TBST) solution and incubated at $4^{\circ} \mathrm{C}$ overnight with anti-PAK5 (catalog no., ab110069; Abcam), anti-MMP2 (catalog no., ab37150; Abcam), anti-epithelial (E-)cadherin (catalog no., ab40772; Abcam), anti-vimentin (catalog no., ab92547; Abcam) and anti-fibronectin (catalog no., ab2413; Abcam) antibody. Following three washes with TBST solution, the membrane was incubated with anti-rabbit horseradish peroxidase-conjugated secondary antibody (dilution, 1:5,000; catalog no., sc-2004; Santa Cruz Biotechnology, Inc., Dallas, TX, USA) at room temperature for $2 \mathrm{~h}$. The signals of the detected proteins were visualized on an Electrochemiluminescence Plus Western Blotting Detection system (GE Healthcare Life Sciences, Chalfont, UK). GAPDH protein levels were used as a control.

Scratch wound healing assay. Cells were grown in 6-well tissue culture dishes until reaching confluence. Cultures were incubated for 10 min with PBS buffer. A scrape was made through the confluent monolayer with a 1-mm diameter plastic pipette tip. Subsequently, dishes were washed twice and incubated at $37^{\circ} \mathrm{C}$ in fresh DMEM (Gibco; Thermo Fisher Scientific, Inc.) containing $10 \%$ FBS. At the bottom of each dish, two arbitrary places were marked where the width of the wound was measured using an IX71 inverted microscope (x4 objective) (Olympus Corporation, Tokyo, Japan). Wound healing was expressed as the mean \pm standard error of the mean (SEM) at 0 and $24 \mathrm{~h}$.

Cell migration assay. Transwell migration assays were performed as previously described (22). Assays were performed using $8-\mu \mathrm{m}$ Transwell inserts (EMD Millipore). Migrated cells were fixed for 30 min with $4 \%$ paraformaldehyde and stained with $0.1 \%$ crystal violet for $20 \mathrm{~min}$. The assays were monitored over a $24-\mathrm{h}$ period. Images of five random fields were captured using an IX71 inverted microscope. The cells were counted in ten random fields using high power at x200 magnification. All experiments were performed in triplicate.

In vivo study. Male BALB/c nude mice ( $\mathrm{n}=3$ per group, between 5 and 6 weeks of age) weighing $\sim 20 \mathrm{~g}$ each were purchased from Shanghai Rubicam Laboratory Animal Ltd. (Shanghai, China) and housed in a controlled environment at $22^{\circ} \mathrm{C}$ with a relative humidity of $65 \%$ and a 12 -h light/12-h dark cycle. All mice had free access to water and food. Non-transduced and transduced Saos-2 cells at $\sim 92 \%$ viability by trypan blue stain assay were subcutaneously injected into the right flank of each mouse $\left(2 \times 10^{7}\right.$ cells/mouse). Solid tumor volumes (in $\mathrm{mm}^{3}$ ) were determined using digital Vernier caliper measurements and the formula $\mathrm{a} \times \mathrm{b}^{2} \mathrm{x} 0.5$, where $\mathrm{a}$ is the longest diameter of the tumor and $b$ is the shortest diameter of the tumor. Following tumor growth for 40 days, the mice were sacrificed and the tumors were excised. All animal procedures were approved by the Animal Ethics Committee of Shanghai Jiao Tong University Affiliated Sixth People's Hospital.

Statistical analysis. For statistical analysis, SPSS software (version 13.0; SPSS Inc., Chicago, IL, USA) was used. All results are presented as the mean \pm standard deviation All results were analyzed using one-way analysis of variance to compare the measurement data with heterogeneity of variance or enumeration data. The Kaplan-Meier estimator method was used to analyze survival. $\mathrm{P}<0.05$ was considered to indicate a statistically significant difference.

\section{Results}

PAK5 overexpression during OS progression. Aberrant PAK5 signaling is integral for breast cancer (23), ovarian cancer (24) and hepatocellular carcinoma cells (25). In the present study, primary samples from 65 patients with OS and 13 pulmonary metastasis samples were analyzed for PAK5 expression using RT-qPCR and IHC (Fig. 1A and B). The clinicopathological characteristics of 65 patients are presented in Table I. PAK5 expression was identified to be significantly associated with clinical stage $(\mathrm{P}=0.035)$, tumor cell necrosis rate $(\mathrm{P}=0.035)$ and metastasis $(\mathrm{P}=0.010)$. No significant differences were identified for other clinical features including sex, age, tumor location and pathological type between individuals with high and low PAK5 expression. Fig. 1A and B indicate that the greatest increase in PAK5 expression occurred in lung metastasis tissue compared with the other tissues. PAK5 expression in tissue from patients with OS with lung metastasis was increased compared with those without lung metastasis. Furthermore, the prognostic significance of PAK5 expression was assessed in 65 patients with OS with clinical follow-up records. Kaplan-Meier estimator analysis of survival demonstrated that the high PAK5 group tended to have significantly shorter overall survival times (Fig. 1C) compared with the low PAK5 group $(\mathrm{P}=0.036)$. 
Table I. Analysis of PAK5 expression and the clinical characteristics of patients with osteosarcoma.

\begin{tabular}{|c|c|c|c|}
\hline Characteristics & $\begin{array}{l}\text { Low level } \\
\text { of PAK5 }\end{array}$ & $\begin{array}{c}\text { High level } \\
\text { of PAK5 }\end{array}$ & P-value \\
\hline Age, years & & & 0.235 \\
\hline$<15$ & 15 & 24 & \\
\hline$\geq 15$ & 13 & 13 & \\
\hline Sex & & & 0.590 \\
\hline Male & 14 & 27 & \\
\hline Female & 14 & 10 & \\
\hline Anatomical location & & & 0.460 \\
\hline Tibia/femur & 17 & 19 & \\
\hline Elsewhere & 11 & 18 & \\
\hline Tumor size, cm & & & 0.095 \\
\hline$\leq 8$ & 18 & 16 & \\
\hline$>8$ & 10 & 21 & \\
\hline Clinical stage & & & 0.035 \\
\hline IIA & 18 & 14 & \\
\hline IIB/III & 10 & 23 & \\
\hline Necrosis rate, $\%$ & & & 0.035 \\
\hline$\leq 25$ & 9 & 19 & \\
\hline$>25$ & 19 & 18 & \\
\hline Pathological type & & & 0.863 \\
\hline Conventional & 18 & 23 & \\
\hline Non-conventional & 10 & 14 & \\
\hline Metastasis & & & 0.010 \\
\hline Negative & 23 & 19 & \\
\hline Positive & 5 & 18 & \\
\hline PAK5 score & 34 & 130 & $<0.001$ \\
\hline
\end{tabular}

PAK5, p21-activated kinase 5. The staining intensity was scored as follows: 0 , staining $<10 \% ; 1,10-25 \% ; 2,26-50 \% ; 3,51-75 \%$; and $4,>75 \%$.

Knockdown of PAK5 inhibits OS cell migration ability and decreases the expression of MMP2. The results of the present study indicated that the PAK5 expression level was associated with metastasis. In order to investigate this association further, PAK 5 was knocked down to determine its function. It was identified that PAK5 shRNA effectively knocked down PAK5 expression at the RNA and protein level. The inhibition ratio was $>75 \%$. Furthermore, certain factors, which were associated with cell migration, including MMP2, E-cadherin, vimentin and fibronectin, were identified. Only MMP2 expression was identified to be significantly decreased at the RNA and protein levels (Fig. 2A and B). Migration is the most important metastasis-associated process. It was investigated whether PAK5 knockdown was able to decrease the migration capability of OS cells using an in vitro scratch wound healing assay and a Transwell migration assay. The results identified that the PAK5 shRNA group markedly decreased the migratory ability (Fig. 2C and D), indicating that PAK5 is a potential migration regulator.
MMP2 overexpression in lung metastasis. The PAK5-early growth response protein 1 (Egr1)-MMP2 signaling pathway contributes to the migration ability in breast cancer and glioma. Therefore, it was analyzed whether MMP2 and PAK5 exhibited the same expression tendencies in OS tissue using IHC, and it was identified that lung metastasis tissue exhibited increased MMP2 expression compared with primary OS tissue (Fig. 3). A marked association was identified between PAK5 and MMP2 expression with a correlation coefficient of 0.821 by Pearson's rank correlation analysis.

PAK5 silencing decreases xenograft growth. The growth rate of subcutaneous xenografts in the PAK5 shRNA group was significantly increased compared with that in the non-transduced MG63 cells. At the fourth week, tumor volume and weight were significantly increased in the two control groups compared with those in the PAK shRNA group (volume, $315.67 \pm 69.15$ and $308.67 \pm 71.43$ vs. $93.33 \pm 16.1 \mathrm{~mm}^{3} ; \mathrm{P}=0.015$; Fig. 4A). PAK5 and MMP2 staining was more intense in the two control group xenografts compared with that in the treated group (Fig. 4B and C).

\section{Discussion}

PAKs are key effectors of the Rho family GTPases that are divided into group I PAKs (PAK1, PAK2 and PAK3) and group II PAKs (PAK4, PAK5 and PAK6). PAK5 exhibits sequence identity with other PAKs in the p21 GTPase-binding and kinase domains. However, it is completely distinct in other regions compared with other PAKs (26). It has been demonstrated that PAK5 functions as a dynamic signaling node in cancer and is central to a variety of cellular functions, including proliferation, apoptosis and migration. PAK5 overexpression has been observed in a number of tumors (9-12,16-20). Metastasis due to tumor progression is the primary cause of the majority of cancer-related mortality, particularly in patients with OS (27). In total, $<80 \%$ of patients with OS exhibit lung metastasis. OS has increased local aggressiveness, and occasionally migrates to the lung and other bone sites with uncommon lymphoid metastasis (28-31). The 5-year overall survival rate in patients with OS and metastases is between 10 and $30 \%(32,33)$. However, the underlying molecular mechanisms of metastasis in OS remain to be further elucidated. Previous research has focused on what facilitates OS cell metastasis (34-36). The focus of the present study was on PAK5, which decreases cell adhesion by promoting cytoskeletal reorganization including filopodia formation and neurite outgrowth (37). The association between PAK5 expression and the outcomes of 65 cases of primary OS tissues was analyzed using RT-qPCR and IHC. A total of 13 lung metastasis tissues were also examined. PAK5 levels were increased in lung metastasis tissues compared with in primary OS tissue. Almost all of the samples exhibited intense positive tan particles with large amounts of cytoplasm. The primary OS tissues were divided into two groups: Low PAK5 and high PAK5. Kaplan-Meier estimator analysis of survival indicated that increased PAK5 expression was associated with a shorter survival time of the patients. No significant differences were identified in certain clinical features including sex, age, tumor location 


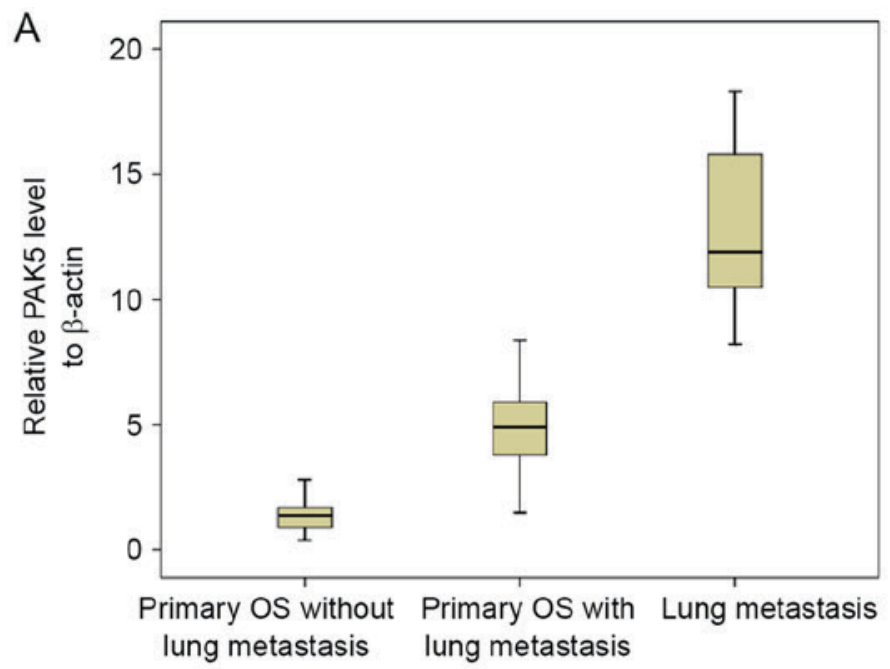

B Primary OS tissue without
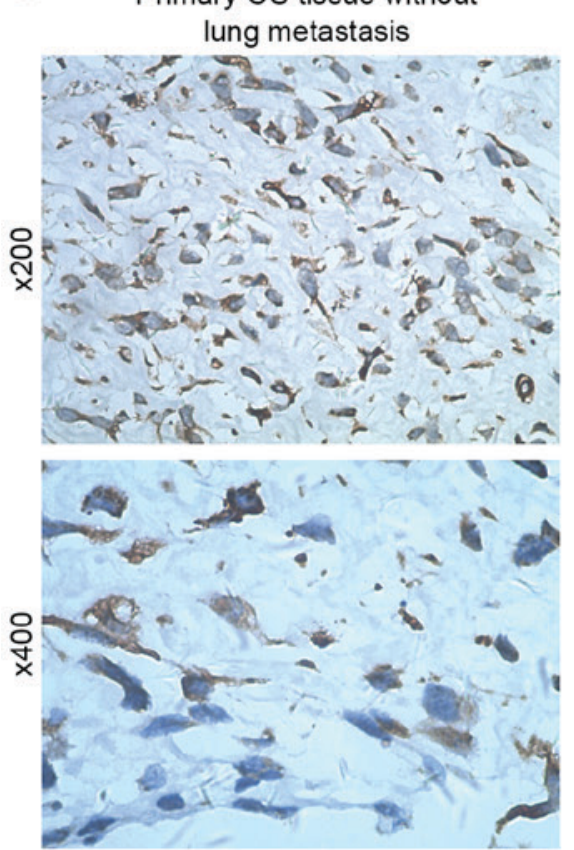

Primary OS tissue with lung metastasis
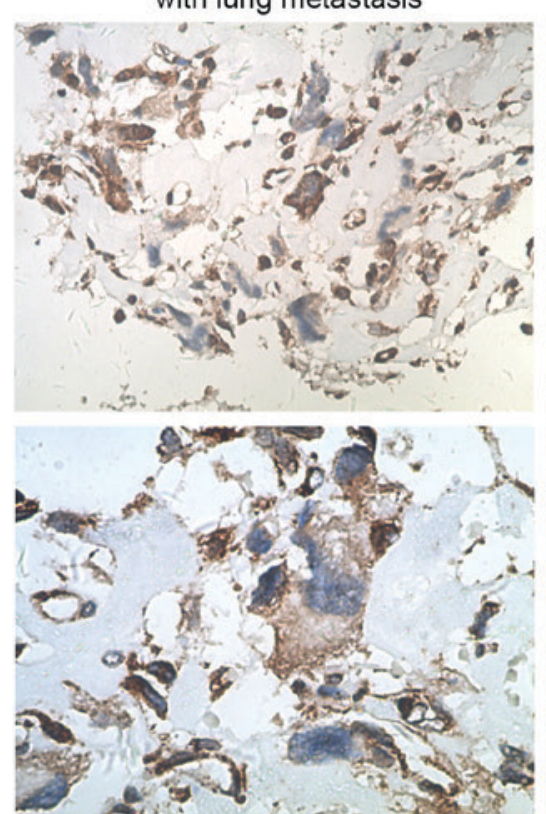

Lung metastasis
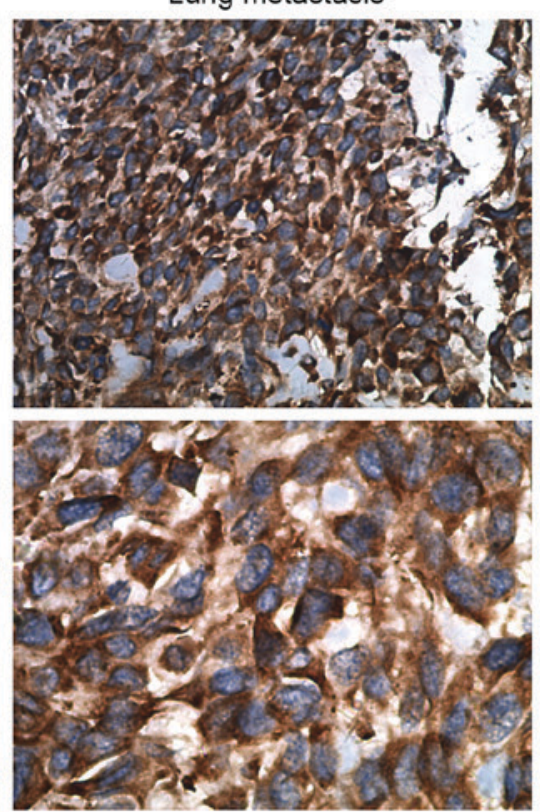

C

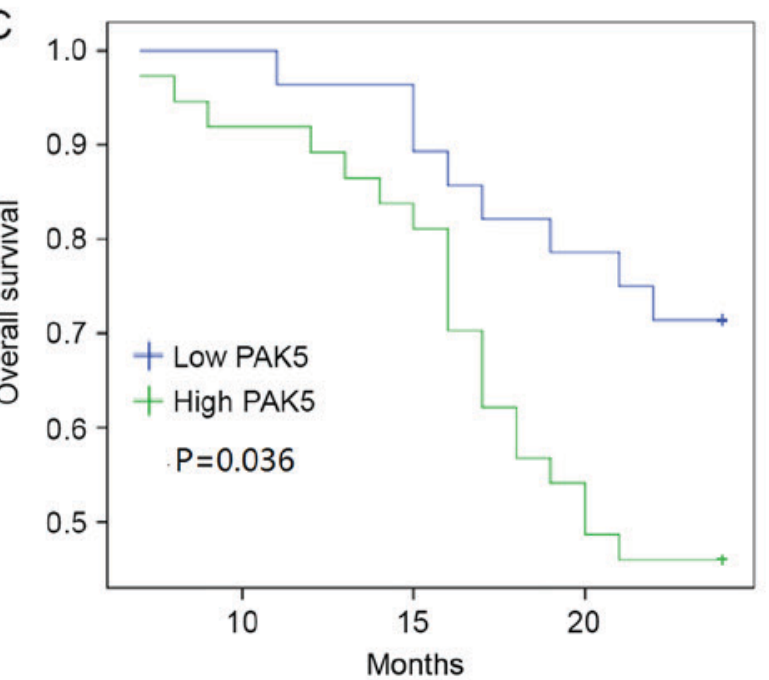

Figure 1. Overexpression of PAK5 in OS lung metastasis tissue. (A) Reverse transcription-quantitative polymerase chain reaction analysis of the PAK5 mRNA transcript levels in 65 primary OS tissue samples and 13 lung metastasis tissues. (B) PAK5 expression levels detected using immunohistochemistry. Upper row, x200 magnification. Lower row, x400 magnification. (C) Kaplan-Meier estimator analysis of survival of 65 patients with OS comparing high and low PAK5 expression ( $\mathrm{P}=0.036)$. PAK5, p21-activated kinase 5; OS, osteosarcoma. 

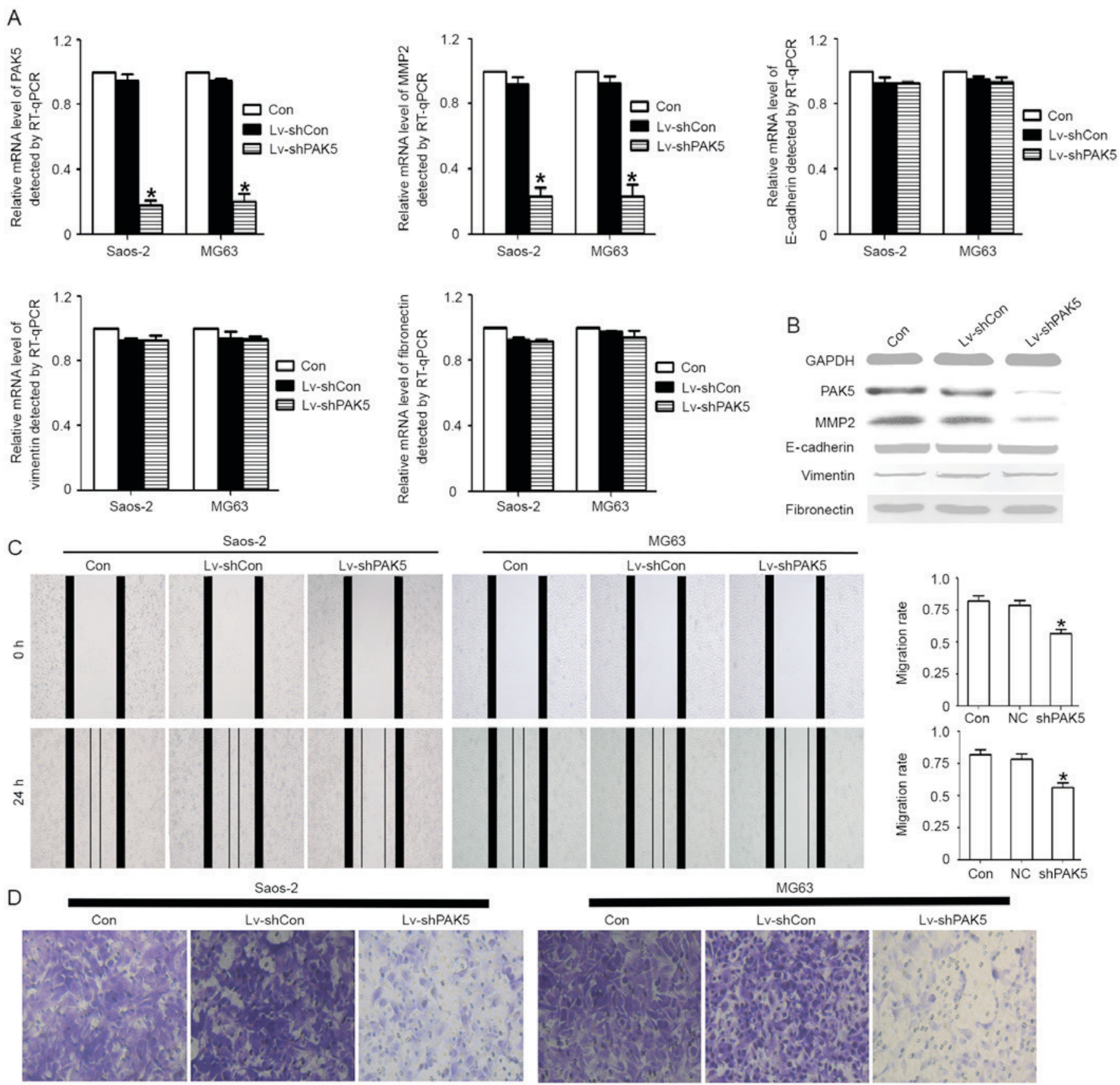

Figure 2. Association between PAK5 and osteosarcoma cell migratory ability. (A) The PAK5 gene was knocked down using Lv-shPAK5 in Saos2 and MG63 cells. The mRNA level of PAK5 and MMP2 was decreased significantly $(>75 \%)$ in the two cell lines ( $\mathrm{P}<0.05)$. No statistically significant difference was identified for E-cadherin, vimentin or fibronectin levels. (B) Western blotting results indicated a marked difference for PAK5 and MMP2 protein expression among these groups. No significant differences were identified for E-cadherin, vimentin and fibronectin. (C) A scratch wound healing assay identified that Lv-shPAK5-transfected Saos2 and MG63 cells exhibited a significantly decreased wound healing rate compared with untreated and Lv-shCon cells. Results are expressed as the mean \pm standard deviation. ${ }^{*} \mathrm{P}<0.05$. (D) An in vitro Transwell migration assay identified that PAK5 knockdown attenuated cell migration. The untreated, Lv-shCon and Lv-shPAK5 cells were seeded in the upper Transwell chambers and incubated for $24 \mathrm{~h}$. Images were captured using a light microscope at x200 magnification of the cells that had migrated into the lower chamber. PAK5, p21-associated kinase 5; Lv-shPAK5, lentivirus-transduced short hairpin RNA targeting PAK5; MMP2, matrix metalloproteinase 2; E-cadherin, epithelial cadherin; Lv-shCon/NC, lentivirus-transduced negative control short hairpin RNA; RT-qPCR, reverse transcription-quantitative polymerase chain reaction; Con, control.

and pathological type between individuals with high and low PAK5 expression. PAK5 expression is associated with clinical stage, necrosis rate and metastasis. In order to investigate the function of PAK5, shRNA was used as an effective way to silence gene expression. The shRNA was able to inhibit PAK 5 expression by $>75 \%$ at the mRNA and protein levels. The downstream factors of PAK5 were also examined. A previous study identified that PAK5 was able to promote metastasis by regulating the Egr1-MMP2 signaling pathway (38). The results of the present study also clarified that, following PAK5 silencing, MMP2, but not E-cadherin, vimentin or fibronectin, was downregulated. MMP2 has been identified as a key extracellular matrix factor that participates in cell migration associated with tumor metastasis (39). PAK5 shRNA decreases the migration ability of OS cells associated with decreased MMP2. The results of the present 


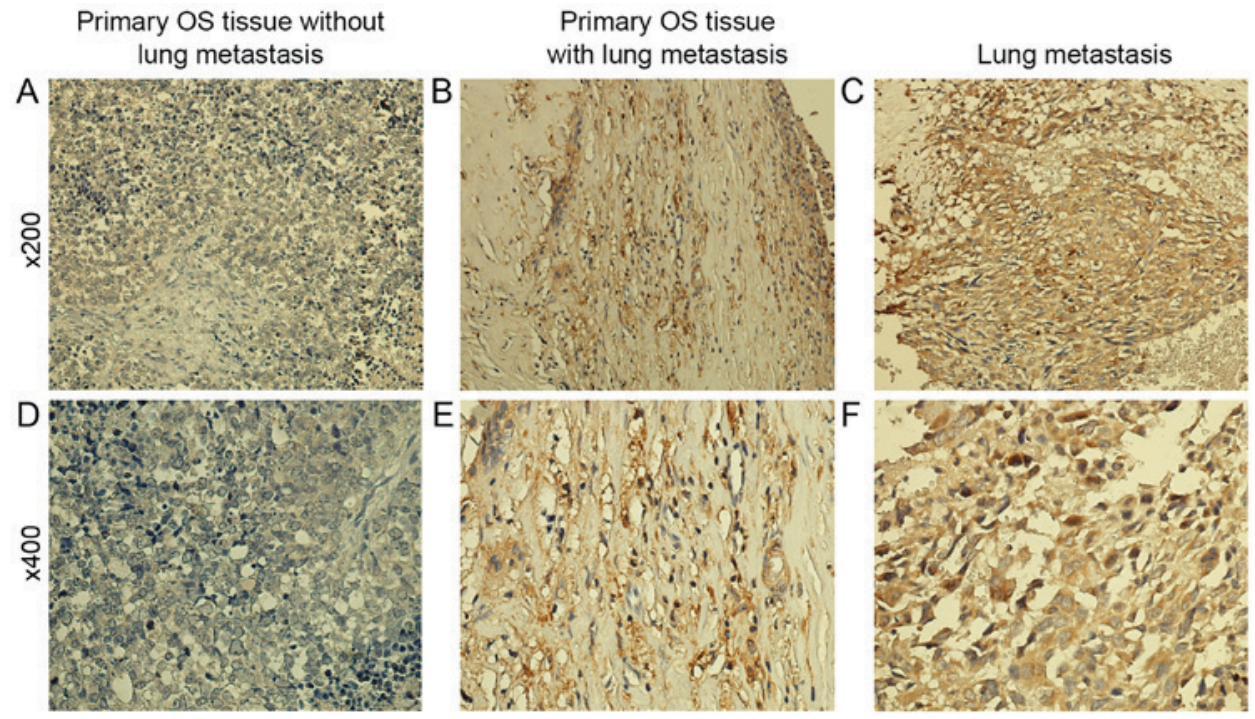

Figure 3. Overexpression of MMP2 in OS lung metastasis tissue. MMP2 expression in 65 primary OS tissue and 13 lung metastasis tissue samples was detected using immunohistochemistry. (A) Primary OS tissue from patients with no metastasis at x200 magnification. (B) Primary OS tissue from patients with lung metastasis at x200 magnification. (C) Lung metastasis tissue at x200 magnification. (D) Primary OS tissue from patients with no metastasis at x400 magnification. (E) Primary OS tissue from patients with lung metastasis at x400 magnification. (F) Lung metastasis tissue at x400 magnification. MMP2, matrix metalloproteinase 2; OS, osteosarcoma.

\section{A}

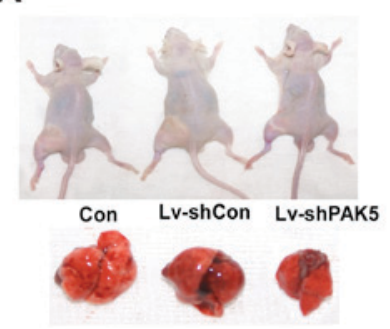

B

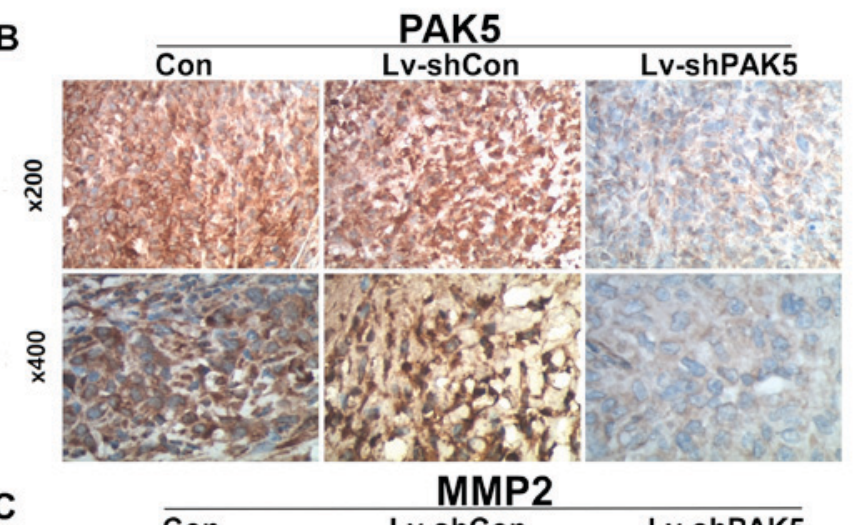

C

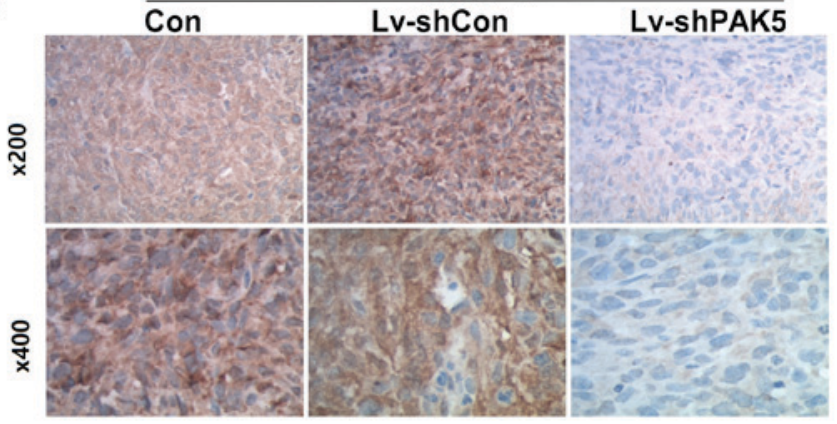

Figure 4. Inhibition of PAK5 inhibits osteosarcoma growth in vitro and decreases MMP2 levels. (A) The Lv-shPAK5 group produced markedly smaller tumors in mice, whereas the untreated and $\mathrm{Lv}$-shCon groups led to the largest tumors in mice $(\mathrm{P}<0.05)$. Tumor volumes are presented in the growth curve. Using immunohistochemistry, it was identified that (B) PAK5 and (C) MMP2 expression were decreased in the Lv-shPAK5 group. PAK5, p21-activated kinase 5; MMP2, matrix metalloproteinase 2; Lv-shPAK5, lentivirus-transduced short hairpin RNA targeting PAK5; Lv-shCon, lentivirus-transduced negative control short hairpin RNA; Con, control. 
study also identified that PAK5 serves an important role in the migration capacity of OS cells via MMP2.

The effects of PAK5 were also investigated in vivo. Following subcutaneous injection of MG63 cells with or without PAK5 shRNA transduction in nude mice bearing OS xenografts, the PAK5 shRNA exhibited superior antitumor efficacy in vivo compared with the two control groups. It is noteworthy that the in vivo PAK5 shRNA treatment led to a significant decrease in tumor growth. Additionally, ex vivo histological analysis revealed that the PAK5 shRNA group exhibited decreased expression of PAK 5 and MMP 2 compared with the control groups.

The results of the present study indicate that PAK5 and MMP2 overexpression are directly associated with metastasis and lead to poor prognosis. This retarded PAK5 shRNA-associated OS cell growth in vitro and in vivo in a nude mouse model indicated that PAK5 regulates MMP2 expression and inhibits tumor metastasis. Therefore, the results of the present study indicate that PAK5 is a potential prognostic indicator for human OS.

\section{Acknowledgements}

The present study was supported by the National Natural Science Foundation of China (grant nos. 81172548 and 8137273).

\section{References}

1. Chi S, Conklin L, Qin J, Meyers P, Huvos AG, Healey JH and Gorlick R: The patterns of relapse in osteosarcoma: The Memorial Sloan-Kettering experience. Pediatr Blood Cancer 42: 46-51, 2004.

2. Crompton BD, Goldsby RE, Weinberg VK, Feren R, O'Donnell RJ and Ablin AR: Survival after recurrence of osteosarcoma: A 20-year experience at a single institution. Pediatr Blood Cancer 47: 255-259, 2006.

3. Gelderblom H, Jinks RC, Sydes M, Bramwell VH, van Glabbeke M, Grimer RJ, Hogendoorn PC, McTiernan A, Lewis IJ, Nooij MA, et al: Survival after recurrent osteosarcoma: Data from 3 European Osteosarcoma Intergroup (EOI) randomized controlled trials. Eur J Cancer 47: 895-902, 2011.

4. Hattinger CM, Pasello M, Ferrari S, Picci P and Serra M: Emerging drugs for high-grade osteosarcoma. Expert Opin Emerg Drugs 15: 615-634, 2010.

5. Ray-Coquard I and Le Cesne A: A role for maintenance therapy in managing sarcoma. Cancer Treat Rev 38: 368-378, 2012.

6. Yang J and Zhang W: New molecular insights into osteosarcoma targeted therapy. Curr Opin Oncol 25: 398-406, 2013.

7. Luetke A, Meyers PA, Lewis I and Juergens H: Osteosarcoma treatment-where do we stand? A state of the art review. Cancer Treat Rev 40: 523-532, 2014.

8. Sells MA and Chernoff J: Emerging from the Pak: The p21-activated protein kinase family. Trends Cell Biol 7: 162-167, 1997.

9. Edwards DC, Sanders LC, Bokoch GM and Gill GN: Activation of LIM-kinase by Pak1 couples Rac/Cdc42 GTPase signalling to actin cytoskeletal dynamics. Nat Cell Biol 1: 253-259, 1999.

10. Marlin JW, Eaton A, Montano GT, Chang YW and Jakobi R: Elevated p21-activated kinase 2 activity results in anchorage independent growth and resistance to anticancer drug-induced cell death. Neoplasia 11: 286-297, 2009.

11. Somanath PR, Vijai J, Kichina JV, Byzova T and Kandel ES The role of PAK-1 in activation of MAP kinase cascade and oncogenic transformation by Akt. Oncogene 28: 2365-2369, 2009.

12. Maroto B, Ye MB, von Lohneysen K, Schnelzer A and Knaus UG: P21-activated kinase is required for mitotic progression and regulates Plk1. Oncogene 27: 4900-4908, 2008.

13. Jaffer ZM and Chernoff J: p21-activated kinases: Three more join the Pak. Int J Biochem Cell Biol 34: 713-717, 2002.
14. Dan C, Nath N, Liberto M and Minden A: PAK5, a new brainspecific kinase, promotes neurite outgrowth in N1E-115 cells. Mol Cell Biol 22: 567-577, 2002.

15. Cotteret S, Jaffer ZM, Beeser A and Chernoff J: p21-activated kinase 5 (Pak5) localizes to mitochondria and inhibits apoptosis by phosphorylating BAD. Mol Cell Biol 23: 5526-5539, 2003.

16. Giroux V, Dagorn JC and Iovanna JL: A review of kinases implicated in pancreatic cancer. Pancreatology 9: 738-754, 2009.

17. Gong W, An Z, Wang Y, Pan X, Fang W, Jiang B and Zhang H: P21-activated kinase 5 is overexpressed during colorectal cancer progression and regulates colorectal carcinoma cell adhesion and migration. Int J Cancer 125: 548-555, 2009.

18. Gu J, Li K, Li M, Wu X, Zhang L, Ding Q, Wu W, Yang J, Mu J, Wen $\mathrm{H}$, et al: A role for $\mathrm{p} 21$-activated kinase 7 in the development of gastric cancer. FEBS J 280: 46-55, 2013.

19. Wang XX, Cheng Q, Zhang SN, Qian HY, Wu JX, Tian H, Pei DS and Zheng JN: PAK5-Egr1-MMP2 signaling controls the migration and invasion in breast cancer cell. Tumor Biol 34: 2721-2729, 2013.

20. Han ZX, Wang XX, Zhang SN, Wu JX, Qian HY, Wen YY, Tian H, Pei DS and Zheng JN: Downregulation of PAK5 inhibits glioma cell migration and invasion potentially through the PAK5-Egr1-MMP2 signaling pathway. Brain Tumor Pathol 31: 234-241, 2014.

21. Livak KJ and Schmittgen TD: Analysis of relative gene expression data using real-time quantitative PCR and the 2(-Delta Delta C(T)) method. Methods 25: 402-408, 2001.

22. Lin F, Shen Z, Tang LN, Zheng SE, Sun YJ, Min DL and Yao Y: KLF8 knockdown suppresses proliferation and invasion in human osteosarcoma cells. Mol Med Rep 9: 1613-1617, 2014.

23. Li Y, Ke Q, Shao Y, Zhu G, Li Y, Geng N, Jin F and Li F: GATA1 induces epithelial-mesenchymal transition in breast cancer cells through PAK5 oncogenic signaling. Oncotarget 6: 4345-4356, 2015.

24. Li D, Yao X and Zhang P: The overexpression of P21-activated kinase 5 (PAK5) promotes paclitaxel-chemoresistance of epithelial ovarian cancer. Mol Cell Biochem 383: 191-199, 2013.

25. Zhai J, Qu S, Li X, Zhong J, Chen X, Qu Z and Wu D: miR-129 suppresses tumor cell growth and invasion by targeting PAK5 in hepatocellular carcinoma. Biochem Biophys Res Commun 464: 161-167, 2015.

26. Pandey A, Dan I, Kristiansen TZ, Watanabe NM, Voldby J, Kajikawa E, Khosravi-Far R, Blagoev B and Mann M: Cloning and characterization of PAK5, a novel member of mammalian p21-activated kinase-II subfamily that is predominantly expressed in brain. Oncogene 21: 3939-3948, 2002.

27. Farfalli GL, Albergo JI, Lobos PA, Smith DE, Streitenberger PD, Pallotta Rodríguez MG and Aponte-Tinao LA: Osteosarcoma lung metastases. Survival after chemotherapy and surgery. Medicina (B Aires) 75: 87-90, 2015 (In Spanish).

28. Poletajew S, Fus L and Wasiutynski A: Current concepts onpathogenesis and biology of metastatic osteosarcoma tumors. Ortop Traumatol Rehabil 13: 537-545, 2011 (In English, Polish).

29. Endo-Munoz L, Evdokiou A and Saunders NA: The role of osteoclasts and tumour-associated macrophages in osteosarcoma metastasis. Biochim Biophys Acta 1826: 434-442, 2012.

30. Friedrich P, Ortiz R, Strait K, Fuentes S, Gamboa Y, Arambú I, Ah-Chu-Sanchez M, London W, Rodríguez-Galindo C and Antillón-Klussmann F: Pediatric sarcoma in Central America: Outcomes, challenges, and plans for improvement. Cancer 119: 871-879, 2013.

31. Rainusso N, Wang LL and Yustein JT: The adolescent and oung adult with cancer: State of the art-bone tumors. Curr Oncol Rep 15: 296-307, 2013.

32. Hiller C, Wegler JL and Forest CP: Osteosarcoma: Accurately diagnosing this bone-chilling disease. JAAPA 29: 29-35, 2016.

33. Meyers PA: Muramyl tripeptide (mifamurtide) for the treatment of osteosarcoma. Expert Rev Anticancer Ther 9: 1035-1049, 2009.

34. Seong BK, Lau J, Adderley T, Kee L, Chaukos D, Pienkowska M, Malkin D, Thorner P and Irwin MS: SATB2 enhances migration and invasion in osteosarcoma by regulating genes involved in cytoskeletal organization. Oncogene 34: 3582-3592, 2015.

35. Lamora A, Talbot J, Bougras G, Amiaud J, Leduc M, Chesneau J, Taurelle J, Stresing V, Le Deley MC, Heymann MF, et al: Overexpression of smad7 blocks primary tumor growth and lung metastasis development in osteosarcoma. Clin Cancer Res 20: 5097-5112, 2014. 
36. Luther GA, Lamplot J, Chen X, Rames R, Wagner ER, Liu X, Parekh A, Huang E, Kim SH, Shen J, et al: IGFBP5 domains exert distinct inhibitory effects on the tumorigenicity and metastasis of human osteosarcoma. Cancer Lett 336: 222-230, 2013.

37. Wong LE, Reynolds AB, Dissanayaka NT and Minden A: p120-catenin is a binding partner and substrate for Group B Pak kinases. J Cell Biochem 110: 1244-1254, 2010.

38. Han ZX, Wang XX, Zhang SN, Wu JX, Qian HY, Wen YY, Tian H, Pei DS and Zheng JN: Downregulation of PAK5 inhibits glioma cell migration and invasion potentially through the PAK 5-Egr1-MMP2 signaling pathway. Brain Tumor Pathol 31: 234-241, 2014.
39. Liu J, Ping W, Zu Y and Sun W: Correlations of lysyl oxidase with MMP2/MMP9 expression and its prognostic value in non-small cell lung cancer. Int J Clin Exp Pathol 7: 6040-6047, 2014.

(i) (\$) $\Theta$ This work is licensed under a Creative Commons Attribution-NonCommercial-NoDerivatives 4.0 International (CC BY-NC-ND 4.0) License. 